\title{
ES HORA DE ACTUAR LA LEGITIMIDAD DEL PODER DE LAS CELEBRIDADES EN EL ÁMBITO DE LAS RELACIONES INTERNACIONALES
}

\author{
TAKE ACTION NOW \\ THE LEGITIMACY OF CELEBRITY POWER \\ IN INTERNATIONAL RELATIONS
}

\section{IL EST TEMPS DE JOUER \\ LA LÉGITIMITÉ DU POUVOIR DES GÉLÉBRITÉS SUR LA SCÈNE INTERNATIONALE}

\author{
Lena PARTzSch \\ Universidad de Friburgo \\ lena.partzsch@ifp.uni-freiburg.de
}

Resumen: Las celebridades son capaces de movilizar un sinnúmero de personas por todo el mundo. En su discurso de aceptación del premio Oscar, Leonardo DiCaprio conminó a su público internacional a trabajar juntos para combatir el cambio climático. Otro ejemplo del activismo de las celebridades son las campañas de Daryl Hannah a favor de los biocombustibles, por una parte, y en contra del oleoducto Keystone XL, por otra. Este artículo analiza la legitimidad del poder de sendas celebridades en el ámbito de las Relaciones Internacionales a la luz de tres criterios, a saber: influencia política, amplia participación, control y rendición de cuentas. Aunque las demandas de las celebridades sigan siendo imprecisas y no prosperen en el Concierto de las Naciones, el poder de éstas puede legitimarse, sin duda, por medio de su influencia política.

Palabras clave: celebridades, cambio climático, políticas ambientales, legitimidad, poder.

Abstract: Celebrities are able to mobilize a wide range of people on a global scale. In his Oscar acceptance speech, Leonardo DiCaprio urged his international audience to work collectively to combat climate change. Another example of celebrity activism is Daryl Hannah's support for biofuels and the 
campaign against the Keystone xL pipeline. The article analyzes the legitimacy of such celebrity power in international relations, along three criteria (political impact; broad participation; control and accountability). It argues that as long as celebrities' claims are vague and do not go further than un consensus, celebrity power can be considered legitimate through the political impact.

Keywords: celebrity, climate change, environmental politics, legitimacy, power.

Traducción de Gonzalo Celorio Morayta

Résumé: Les célébrités sont capables de mobiliser un grand nombre de personnes autour du monde. Au moment d'accepter un Oscar, dans son discours Leonardo Di Caprio a invité le public international à travailler tous ensemble contre le changement climatique. On peut citer aussi, comme exemple d'activisme des célébrités, la campagne de Daryl Hannah en faveur des biocombustibles et contre l'oléoduc Keystone xL. Cet article s'interroge sur la légitimité du pouvoir qu'ont exercé ces deux personnages dans le domaine international, selon trois indicateurs: l'influence politique, l'ampleur de la participation, et le contrôle et la présentation des comptes. Même si les exigences des célébrités ne sont toujours pas précises et si le concert des nations ne les couronne guère de succès, elles ont un pouvoir dont la légitimité découle de leur influence politique.

Mots clefs: célébrité, changement climatique, politiques pour l'environnement, légitimité, pouvoir.

Traducción de Bernardo MabiRe

Fecha de recepción: diciembre 2016

Fecha de aceptación: abril de 2017 
$\mathrm{E}$ N EL ÁMBITo de las Relaciones Internacionales, * las celebridades adquieren cada vez mayor poder político y muchas de ellas están comprometidas con causas medioambientales. Con frecuencia mobilizan a una amplia gama de personas e instan a los gobiernos a actuar en temas como el de la política climática. En 2016, durante su discurso de aceptación del Oscar por su papel en The Revenant, Leonardo DiCarpio exhortó a su público internacional a trabajar de manera colectiva y a dejar de aplazar la acción en contra del cambio climático. ${ }^{1}$ Otro ejemplo es la actriz estadounidense Daryl Hannah. Poco antes de la Cumbre del Clima de París en 2015, el presidente de los Estados Unidos, Barack Obama, anunció el rechazo de su gobierno al oleoducto Keystone xL, en contra del cual Hannah había hecho campaña durante varios años. ${ }^{2}$ Este artículo analiza, por un lado, si este poder que las celebridades ostentan es legítimo o no y, por otro, cómo pueden aumentar o menoscabar la toma de decisiones más allá del Estado-nación.

Una de las tareas políticas más complejas a las que nos enfrentamos es alcanzar la democracia en el ámbito global. Pese a no ser democrática, la Organización de las Naciones Unidas (ONU) ofrece un orden internacional que, bajo el principio de "un país, un voto", es el que más se acerca a las normas democráticas. En él, las intervenciones políticas de actores no estatales, como las celebridades no electas, sólo se consideran legítimas cuando han sido sancionadas por sus propios Estados o por algún organismo internacional. Éste es el caso de los embajadores de buena voluntad de la onU, como DiCaprio mismo. Sin embargo, la mayoría de las celebridades -como sucede con Hannah- no cuenta con una autorización formal. Se puede argumentar que las celebridades

* Se publica el artículo que aquí se ofrece por el acuerdo establecido entre Global Governance y Foro Internacional, según el cual ésta incluirá entre los propios un artículo de aquélla cada año y viceversa.

1 "2016 Oscar acceptance speech for best actor", 2016, en www.oscar. go.com/news/winners.

2 "Keystone XL Pipeline key events from proposal to Obama's rejection”, Huffington Post, 2016, en www.huffingtonpost.ca. 
no dejan de ser ciudadanos individuales que en la arena de las Relaciones Internacionales hablan y actúan únicamente por sí mismos. Aún más: podría decirse que representan el ideal cosmopolita de "ciudadano del mundo". 3 Aun cuando en su calidad de ciudadanos (del mundo) pueden sustentar sus hechos en la libertad de expresión que la Constitución garantiza, las celebridades no son ciudadanos comunes. El aumento de su poder se arraiga en las nuevas formas de gobernanza y en la creciente relevancia de la autoridad privada en la política global. ${ }^{4}$

Ya que las celebridades, por lo menos en algunos casos, suelen influir no poco en las Relaciones Internacionales, ${ }^{5}$ debemos cuestionar la legitimidad de su poder. Por "poder de las celebridades" me refiero a su "capacidad... para determinar las acciones e incluso las ideas de los demás". ${ }^{6}$ En la primera sección de este artículo, se hará un esbozo del poder de las celebridades en el ámbito de las Relaciones Internacionales y se presentarán, de manera detallada, los dos casos ilustrativos en comento (DiCaprio y Hannah). Esto nos lleva a la segunda sección en la que, con base en la bibliografía sobre la legitimidad de los actores no estatales en la gobernanza global, se desarrollarán tres criterios que permitan evaluar tal legitimidad: 1) legitimidad por influencia política; 2) legitimidad por amplia participación del demos (total de personas

${ }^{3}$ T. Stein, "Global social and civil entrepreneurs: An answer to the poor performance of global governance?", Wissenschaftszentrum Berlin, 2012, en www.bibliothek.wzb.eu.

${ }^{4}$ L. A. Richey y S. Ponte, "Better (Red) ${ }^{\mathrm{TM}}$ than dead? Celebrities, consumption and international aid", Third World Quaterly, vol. 29, núm. 4, 2008, pp. 711-729.

${ }^{5}$ Richey y A. C. Budabin, "Celebritizing conflict: How Ben Affleck sells the Congo to American", Humanity: An International Journal of Human Rights, Humanitarianism, and Development, vol. 7, núm. 1, 2016, pp. 27-46; Richey, Celebrity humanitarianism and North-South relations: Politics, place and power, Nueva York, Routledge, 2016; y M. Wheeler, Celebrity politics: image and identity in contemporary political communications, Cambridge, Polity Press, 2013.

${ }^{6}$ L. Partzsch, "The power of celebrities in global politics", Celebrity Studies, vol. 6, núm. 2, 2015, p. 183. 
afectadas y ciudadanos potenciales); 3) legitimidad por control y rendición de cuentas. Estos criterios reflejan dimensiones distintas e interrelacionadas del proceso democrático de toma de decisiones. Las interrelaciones se explicarán cuando se introduzcan los criterios, los que se utilizarán para analizar, en la tercera sección del artículo, la legitimidad de los casos de DiCaprio y Hannah. Metodológicamente, se utilizaron los tres criterios como códigos y una serie de preguntas (véase cuadro 1) como subcódigos para analizar un total de noventa documentos de la última década (abril de 2006 a marzo de 2016): artículos sobre las dos celebridades, entrevistas que hayan concedido o discursos que hayan pronunciado. La búsqueda de textos se hizo por medio de Internet y no incluye ninguna noticia de menos de cinco oraciones.

Sostengo que mientras las demandas de las celebridades sean muy vagas y no trasciendan el consenso de las Naciones Unidas, se puede considerar que su poder es legítimo por la influencia política que conlleva. Si bien es cierto que DiCaprio y Hannah contribuyen a la agenda internacional con sus perspectivas de sociedad civil, no necesariamente dan voz a las posturas más marginalizadas y, por tanto, su legitimidad democrática según la amplitud de la participación del demos en las Relaciones Internacionales es ambivalente. Finalmente, al instar a los gobiernos a que cumplan con los acuerdos internacionales, como lo hace DiCaprio, los responsabiliza en nombre de la ciudadanía para que rindan cuentas. Sin embargo, no hay un mecanismo para que las celebridades rindan cuentas de su actuación política ante las personas afectadas a las que dicen representar. Esta falta de control representa un problema, cuando transmiten posicionamientos más radicales, que generalmente no cuentan con el apoyo de la comunidad internacional. Ése es el caso de las protestas de Hannah en contra del Keystone XL y de su promoción de los biocombustibles. Sin embargo, el hecho de que este tipo de compromiso problemático pueda servir para hacer frente a los problemas medioambientales y combatir el calentamiento global es otro asunto. 


\section{El PODER DE LAS CELEBRIDADES}

\section{EN LAS RELACIONES INTERNACIONALES}

Las celebridades son personas que gozan, por sobre las demás, de un perfil público prominente. Cuando se involucran en temas de política exterior, suelen utilizar el método clásico de defensoría política. Su popularidad les permite tener acceso a la diplomacia internacional. Cooper los identifica como "celebridades diplomáticas"7 (un diplomático es un funcionario que representa a un Estado para ejercitar la diplomacia en otro Estado o en una organización internacional). Aunque la ONU ha recurrido a celebridades para atraer la atención pública sobre sus campañas desde la década de 1950, Kofi Annan, durante su periodo al frente de la Secretaría General, empezó a incluirlas con mayor frecuencia en las cruzadas de la Organización. ${ }^{8}$ Por tanto, la presencia de celebridades en las Relaciones Internacionales ha aumentado significativamente a lo largo de los últimos quince años. ${ }^{9}$ Un segmento amplio de la población ha notado el compromiso de éstas con distintas causas -como el cambio climático-, y su trabajo de defensoría ha sido acogido en el ámbito internacional.

En julio de 2016, la página electrónica www.looktothestars. org publicó una lista de 837 celebridades comprometidas con la causa medioambiental, ${ }^{10}$ cuya lista encabeza el actor estadounidense Leonardo DiCaprio, quien creó en 1998 la fundación que lleva su nombre y participó en varias películas que buscan educar al público en temas ambientales, como Blood Diamond (2006), The 11th Hour (2007) y The Revenant (2015). ${ }^{11}$

${ }^{7}$ Celebrity Diplomacy, Colorado, Paradigm, 2008.

8 Ibidem, p. 16.

${ }^{9}$ Cf. Partzsch, art. cit.; Richey, op. cit.; y L. Tsaliki, C. Frangonikolopoulos y A. Huliaras (eds.), Transnational celebrity activism in global politics: Changing the world?, Chicago, University of Chicago Press, 2011.

10 "Jennifer Lopez presents UNICEF Women of Compassion Award", 30 de noviembre de 2015, en www.looktothestars.org/news.

11 "Leonardo DiCaprio. Charity Work, Events and Causes", 10 de julio de 2017, en www.looktothestars.org. 
En 2014, DiCaprio se convirtió oficialmente en mensajero de la paz de las Naciones Unidas en contra del cambio climático y el discurso que pronunció en el marco de la Cumbre sobre el Clima de la ONU, celebrada en Nueva York en septiembre de ese mismo año, obtuvo más menciones en titulares que la intervención de cualquier otro jefe de Estado. ${ }^{12}$ En la lista de las cien celebridades más influyentes del mundo que publica Forbes cada año con base en los ingresos obtenidos de la industria del entretenimiento y en la visibilidad de estos personajes en los medios de comunicación, DiCaprio ocupa el lugar octogésimo noveno con un ingreso anual de 89 millones de dólares estadounidenses. ${ }^{13} \mathrm{El}$ actor fue seleccionado como un caso de estudio ilustrativo, porque figura en ambas listas.

El segundo caso de estudio es la primera mujer que apareció en la lista de looktothestars.org, ${ }^{14}$ es decir la actriz de cine estadounidense Daryl Hannah, quien ocupa el lugar número cuatro en el rubro de causa medioambiental luego de Edward Norton, Jack Johnson y DiCaprio. Se la conoce por sus actuaciones en películas como Blade Runner (1982), Wall Street (1987) y Kill Bill (2003). Hannah ha sido arrestada en repetidas ocasiones por su activismo, particularmente por su lucha en contra del oleoducto Keystone XL, que TransCanada Corporation intentó construir entre las arenas bituminosas de Alberta, en Canadá, y las refinerías de Texas, en Estados Unidos. ${ }^{15}$

12 Cf. "Leonardo DiCaprio at the un: «Climate change is not hysteria -it's a fact»”, 2015, The Guardian, en www.theguardian.com, consultado el 30.I.2015.

13 Cf. "The World's Highest- Paid Celebrities", Forbes Magazine, en www.forbes.com.

${ }^{14}$ Look to the Stars es la página electrónica con mayor información acerca de las acciones sociales y compromisos políticos de las celebridades. Los medios de comunicación establecidos (BвC, Associated Press, etc.), los seguidores y el sector no lucrativo recurren a esta página para información. La "lista de las principales celebridades" se basa en una serie de causas y organizaciones benéficas con que las celebridades están comprometidas, así como en los artículos periodísticos que se publican sobre ellas.

15 J. Ostroff, "Daryl Hannah talks Keystone Pipeline, getting arrested, her new doc and Stephen Harper at sxsw", Huffington Post, 14 de marzo de 2014, en http://www.huffingtonpost.ca, consultado el 30.XI.2015. 
Además, Hannah dirige una tienda en línea de productos ecológicos que van desde juguetes para niños hasta vehículos de biodiesel. ${ }^{16}$ También es cofundadora de la Alianza por un Biodiesel Sostenible, cuyo propósito consiste en "promover las prácticas a favor de un biodiesel sustentable y facilitar la comunicación para un futuro de energía sostenible que beneficie a todos los sectores de la sociedad". ${ }^{17}$

Los casos de DiCaprio y Hannah ilustran claramente cómo se entrelazan, en el campo de su activismo ecologista, los ámbitos público y privado de la vida de las celebridades. Una vinculación estratégica entre el entretenimiento, la actividad empresarial y el compromiso con causas benéficas les permite maximizar su publicidad. ${ }^{18}$ Hay diversos estudios que han evaluado las motivaciones personales y la influencia de las celebridades en el terreno de las relaciones internacionales. ${ }^{19}$ Por ejemplo, Andersen evalúa la influencia que tuvo la participación de celebridades en las actividades de Greenpeace en el marco de la Cumbre Río+20, celebrada en 2012. La autora observa que, al recurrir a ellas, la organización aumentó exitosamente la atención de los medios de comunicación sobre las causas medioambientales. Sin embargo, advierte que Greenpeace se arriesga a que su verdadero mensaje se diluya y desdibuje. ${ }^{20} \mathrm{Al}$ ocultar las relaciones sociales y medioambientales del comercio y la producción que apun-

${ }^{16}$ Cf. "Homepage DH Love Life", 2016, en www.dhlovelife.com, consultado el 15.VI.2016.

17 "Daryl Hannah. Charity Work, Events and Causes", en www.looktothestars.org/celebrity/daryl-hannah. También véase "Daryl Hannah does Bio Diesel!", 2016, en www.youtube.com/watch?v=pUZUfRJD0aQ.

18 Cooper, op. cit.

${ }^{19}$ Verbigracia, B. Clifford, The Marketing of rebellion: Insurgents, media, and international activism, Cambridge, University Press, 2005; Richey y Budabin, art. cit.; y L. Chouliaraki, "The theatricality of humanitarianism: A critique of celebrity advocacy", Communication and Critical/Cultural Studies, vol. 9, núm. 1, 2011, pp. 1-21.

20 "«Together we can save the Arctic»: Celebrity advocacy and the Rio Earth Summit 2012”, Celebrity Studies, vol. 3, núm. 4, 2013, pp. 339-352, doi:10.1080/19392397.2013.831617. 
talan la pobreza, la inequidad y la devastación del medio ambiente, es probable que las celebridades contribuyan a aumentar los problemas reales antes que a resolverlos. ${ }^{21}$

Por lo que toca a la influencia política, es de observar que generalmente las celebridades son individuos no electos que se vuelven más influyentes cuando participan en redes transnacionales más amplias. Aunque principalmente intentan influir en los procesos políticos por medio de la defensoría, también instan al público a comprar ciertos productos y boicotear otros. Por ejemplo, Hannah exige, por un lado, que el gobierno estadounidense rechace la ampliación de oleoductos y pide a los consumidores, por otro, que compren combustibles de biomasa y no de petróleo. ${ }^{22}$ Cuando se nos presentan casos de celebridades que hacen trabajo de defensoría, normalmente sus actividades parecen estar a favor de objetivos universales. ${ }^{23}$ Parece, por tanto, que las celebridades realizan buenas acciones, actúan a favor del bien común y permiten que temas globales, como el cambio climático y el bienestar animal, se consideren asuntos relevantes e importantes, a los que se debe hacer frente de manera urgente y que gocen, por tanto, de aceptación. Las celebridades, como DiCaprio y Hannah, se perciben como catalizadores en el apoyo de proyectos y para unir a personas de distintos sectores, como empresas internacionales y organizaciones no gubernamentales. La tendencia dicta que los actos para enfrentar el cambio climático y rechazar el propuesto oleoducto Keystone XL, que hubiera llevado petróleo crudo pesado y contribuido al calentamiento global con sus respectivas emisiones, procuran el bienestar de la población.

Pero a la vez, las celebridades contribuyen también a impulsar ciertas ideas en contra de otras alternativas. Ejercen el poder tanto cooperativamente, como en contra y por sobre los

${ }^{21}$ H. Dieter y R. Kumar, "The downside of celebrity diplomacy: The neglected complexity of Development", Global Governance, vol. 14, núm. 3, 2008, pp. 259-264; y Richey y Ponte, art. cit.

${ }^{22}$ Cf. "Homepage...".

${ }^{23}$ Véase, por ejemplo, Cooper, op. cit., pp. 36-51. 
demás, cuando de coercer y manipular se trata. ${ }^{24}$ Las celebridades determinan agendas públicas y desvían la atención de temas que compiten entre sí. ${ }^{25}$ La agenda climática de DiCaprio compite con otros temas de la política global. El actor propugna por tasar el carbón y fomentar el ecoturismo, lo que ha suscitado no poca controversia; ${ }^{26}$ Hannah, por usar un biodiesel "sostenible" en vez de petróleo, aunque muchos ecologistas adviertan sobre los efectos devastadores que los biocombustibles tienen en la pobreza mundial, la seguridad alimentaria y el cambio del uso de suelo en el Sur global. ${ }^{27}$ Estos ejemplos demuestran que las celebridades toman decisiones políticas, cuando establecen sus agendas públicas, las cuales influyen por lo común pertinentemente, en el caso de que las celebridades sean exitosas, y de ahí que sea menester legitimarlas. ${ }^{28}$

\section{Criterios PARA EVAlUAR LA LEgItimidad \\ DEL PODER DE LAS GELEBRIDADES}

En la década de 1990, la transferencia de competencias que tradicionalmente recaían en los Estados-nación a organizaciones intergubernamentales avivó el debate sobre la legitimidad de una gobernanza que trascendiera el Estado-nación. ${ }^{29}$

24 Partzsch, art. cit.

25 Richey y Budabin, art. cit.

${ }^{26}$ Véase, al respecto, D. J. McCauley, "Selling out nature: Commentary", $\mathrm{Na}$ ture Climate Change, vol.443, núm. 7107, 2006, pp. 27-28, doi:10.1038/443027a.

${ }^{27}$ F. Sengers, R.P.J.M. Raven y A. Van Venrooij, "From riches to rags: Biofuels, media discourses, and resistance to sustainable energy technologies", Energy Policy, vol. 38, núm. 9, 2010, pp. 5013-5027.

28 Cf. F. Nullmeier, A. Geis y C. Daase, "Der Aufstieg der Legitimitätspolitik: Rechtfertigung und Kritik politisch-ökonomischer Ordnungen”, Leviathan Sonderheft, vol. 40, núm. 27, 2012, pp. 9-39; y K. D. Wolf, “Contextualizing normative standards for legitimate governance beyond the state", en R. J. Grote y B. Gbiki (eds.), Participatory Governance. Political and Societal Implications, Opladen, Leske \& Budrich, 2002.

${ }^{29}$ F. W. Scharpf, Governing in Europe: Effective and democratic?, Oxford, University Press, 1999. 
La brecha entre los ciudadanos y los órganos de toma de decisiones en el ámbito internacional era cada vez mayor en un creciente número de campos, entre ellos el de la política medioambiental. ${ }^{30}$ Ante esta situación, la participación de actores no estatales en la política internacional se consideró un mecanismo pragmático para reducir esta distancia, como lo ejemplifican las reuniones anuales de la Convención Marco de las Naciones Unidas sobre el Cambio Climático. ${ }^{31}$ En particular, se tiene a las ONGs por representantes de la sociedad civil y contrapeso del poder, cada vez mayor, del sector privado en la gobernanza global. ${ }^{32}$ Sin embargo, al mismo tiempo aparecieron estudios que cuestionan la legitimidad de las ongs, porque "no son resultado del voto popular". ${ }^{33}$ Además, los expertos han demostrado que la participación de las ONGs depende y produce formas peculiares de subjetividad que las caracterizan. Mientras que algunas de estas formas se tratan como normales, gozan de credibilidad y son aceptables en el terreno de las relaciones internacionales, otras han sido juzgadas indeseables e inaceptables. ${ }^{34}$ Sólo grupos interesados y bien organizados, principalmente de países anglosajones, logran participar en las relaciones internacionales; por ejem-

${ }^{30}$ Wolf, art. cit.

${ }^{31}$ J. Neyer, "Die Stärke deliberativer politischer Theorien und das Elend der orthodoxen Demokratietheorie: Eine Replik auf Marcus Höreth", Zeitschrift für Politikwissenschaft, vol. 19, núm. 3, 2009, pp. 331-358.

${ }^{32}$ Cf. A. Brunnengräber, K. Ansgar y H. Walk (eds.), NGOs als Legitimationsressource: Zivilgesellschaftliche Partizipationsformen im Globalisierungsprozess, Opladen, Leske \& Budrich, 2001; y D. Fuchs, "Commanding Heights? The Strength and Fragility of Business Power in Global Politics”, Millennium: Journal of International Studies, vol. 33, núm. 3, 2005, pp. 771-801.

${ }^{33}$ T. Gebauer, “«...von niemandem gewählt!»: Über die demokratische Legitimation von NGo", en U. Brand, A. Demirovic, C. Görg y J. Hirsch (eds.), Nichtregierungsorganisationen in der Transformation des Staates, Münster, Westfälisches Dampfboot, 2001.

${ }^{34} \mathrm{~K}$. Tucker, "Participation and subjectification in global governance: NGOs, acceptable subjectivities and the wTO", Millenium: Journal of International Studies, vol. 42, núm. 2, 2014, pp. 376-396. 
plo, en las reuniones de la onU. ${ }^{35}$ Hay estudiosos que a este propósito argumentan que las celebridades defensoras de alguna causa podrían paralizar e incluso restar facultades a las personas afectadas, especialmente en el Sur global. ${ }^{36}$

Los debates correspondientes sobre la legitimidad de actores no estatales en el campo de las Relaciones Internacionales cuestionan cómo se justifica el poder de los agentes sobre otras personas y por qué la gente reconoce y acepta su autoridad. ${ }^{37}$ En un caso ideal de legítima toma de decisiones, hay procesos justos basados en normas democráticas que cuentan con una amplia participación del demos (legitimidad orientada a la aportación) y que conducen a decisiones que garantizan la provisión eficaz y justa de servicios (legitimidad orientada a resultados por influencia política). ${ }^{38}$ Sin embargo, sea como fuere el motivo, si se necesita adaptar alguna decisión, también hay procedimientos para revisarla o revocarla (legitimidad orientada al rendimiento por control y rendición de cuentas). Esta tercera dimensión de la legitimidad empieza con los resultados políticos independientemente del proceso y requiere procedimientos adicionales de control y rendición de cuentas. Las personas afectadas por las decisiones (los ciudadanos) deben tener control sobre los actores que dan resultados políticos en su nombre (ya gobiernos, ya actores no estatales). ${ }^{39} \mathrm{Si}$ se analiza la legitimidad del poder de los agentes, su ejercicio generalmente se sustenta en el pasado y en decisiones tomadas con anterioridad. Los acadé-

${ }^{35}$ Cf. M. Kaldor, "The idea of global civil society", International Affairs, vol. 79, núm. 3, 2003, pp. 583-593; y L. Macdonald, "Globalizing civil society: Interpreting international NGOs in Central America", Millennium: Journal of International Studies, vol. 23, núm. 2, 1994, pp. 267-285.

${ }^{36}$ Por ejemplo, Dieter y Kumar, art. cit.

${ }^{37}$ M. Zürn, "Autorität und Legitimität in der postnationalen Konstellation", Leviathan Sonderheft, vol. 40, núm. 27, 2012, pp. 41-62.

${ }^{38}$ Scharpf, op. cit.

${ }^{39}$ I. Take, "Legitimes Regieren auf drei Ebenen - Konzeption und Analyseraster", en I. Take (ed.), Legitimes Regieren jenseits des Nationalstaats: Unterschiedliche Formen von Global Governance im Vergleich, Baden-Baden, Nomos, 2009. 
micos sostienen que así sucede particularmente cuando se trata de justificar el poder de actores no estatales con base en su influencia política; por ejemplo, una vez que las celebridades han recaudado fondos para combatir la pobreza o movilizado a personas de manera eficaz en contra del calentamiento global. Por tanto, en la siguiente sección, empiezo por explicar esta dimensión de la legitimidad y presento tres criterios de la bibliografía sobre la legitimidad de actores no estatales en la gobernanza global: 1) legitimidad por influencia política; 2) legitimidad por participación amplia del demos; 3) legitimidad por control y rendición de cuentas (véase cuadro 1). En principio, se definen estos criterios de forma abstracta y se explica la interrelación entre ellos. Después se utilizan para analizar la legitimidad del poder de las celebridades en la política global. Las restricciones que imponen estos tres criterios no pretenden ignorar la diversidad de normas sobre cómo legitimar el poder político. Se trata, más bien, de un intento por estructurar distintos argumentos y hacerlos operativos para un estudio empírico.

\section{Legitimidad por influencia política}

Con frecuencia, la participación de actores no estatales se considera una oportunidad para solucionar problemas que los gobiernos han demostrado ser incapaces de manejar solos. ${ }^{40} \mathrm{La}$ ONU y otras organizaciones internacionales fracasan repetidamente, cuando atienden problemas urgentes como la pobreza global y el cambio climático. Entre los años más calurosos que se hayan registrado, 2016 encabeza la lista. ${ }^{41}$ La mayoría de los expertos a favor de nuevas formas de gobierno, en las que están involucrados actores no estatales, considera que en la política global la intervención de éstos es legítima, toda

${ }^{40}$ Idem. Véase, además, Wolf, art. cit.

${ }^{41}$ NASA, "Global Climate Change", 2017, en www.climate.nasa.gov, consultado el 20.III.2017. 
vez que son capaces de hacer frente de manera más eficaz a problemas de interés público. ${ }^{42}$

\section{CuAdro 1}

Análisis de la legitimidad de las celebridades en el campo de las Relaciones Internacionales

\begin{tabular}{|c|c|}
\hline Criterio de legitimidad & Preguntas para el análisis empírico \\
\hline 1) Influencia política & $\begin{array}{l}\text { ¿Las celebridades enfrentan eficazmente las inquietudes de la } \\
\text { ciudadanía? ¿Contribuyen a la implementación de objetivos } \\
\text { universales (según la definición de la oNu)? ¿Hay conflictos } \\
\text { políticos (visibles, ocultos o invisibles) en relación con medidas } \\
\text { y objetivos específicos? }\end{array}$ \\
\hline $\begin{array}{l}\text { 2) Amplia participación del } \\
\text { demos }\end{array}$ & $\begin{array}{l}\text { ¿Las celebridades contribuyen a que haya una representación } \\
\text { más equilibrada de todos los intereses y perspectivas en el } \\
\text { campo de las Relaciones Internacionales? }\end{array}$ \\
\hline $\begin{array}{l}\text { 3) Control y rendición de } \\
\text { cuentas }^{\mathrm{a}}\end{array}$ & $\begin{array}{l}\text { ¿Las celebridades ayudan a las personas afectadas (ciudadanos) } \\
\text { para que quienes toman las decisiones rindan cuentas? } \\
\text { ¿Las personas afectadas tienen el derecho de exigir a las cele- } \\
\text { bridades transparencia e información? } \\
\text { ¿Las personas afectadas tienen mecanismos de procuración } \\
\text { para sancionar o premiar ciertas conductas? ¿Hay control so- } \\
\text { cial, como señalar y denunciar? } \\
\text { ¿Las celebridades adaptan su comportamiento como reacción } \\
\text { a las personas afectadas? ¿Se pueden observar cambios de com- } \\
\text { portamiento en respuesta a las críticas o como resultado del } \\
\text { aprendizaje? }\end{array}$ \\
\hline
\end{tabular}

a Véase infra, cuadro 2.

¿Qué significa esto en lo que atañe a la legitimidad del poder de las celebridades? Según este criterio, si las celebridades determinan las acciones e incluso las ideas de otros, ejercen el poder legítimamente cuando se requiere actuar para

42 J. M. Witte y W. Reinicke, Business as unusual: Facilitating United Nations reform through partnerships, Nueva York, United Nations Global Compact Office, 2005. 
resolver problemas urgentes e implementar objetivos universales. Puede darse el caso de que sea imperativo proceder en contra de la voluntad de ciertos actores o grupos particulares para, por ejemplo, detener inmediatamente alguna violación a los derechos humanos o un daño al medio ambiente. En consecuencia, la defensoría por parte de las celebridades es legítima a condición de que persiga cualquier objetivo social o ambiental. Los estudios sobre las celebridades suelen definir el bien común como el antónimo del interés propio de las mismas celebridades. ${ }^{43}$ Con frecuencia, los casos aislados de mejores prácticas que muestran cómo alcanzar metas sociales específicas se convierten en discursos con una carga emocional importante, que instan a individuos responsables a actuar del mismo modo y ser solidarios con quienes ejercen el poder y actúan por terceros. ${ }^{44}$ Entre estos tipos de discurso, cualquier oposición a las supuestas metas sociales debe considerarse ilegítima.

Sin embargo, que las acciones de las celebridades se den por sentado y nadie se oponga a ellas no significa que no haya conflictos ni alternativas, ${ }^{45}$ pues éstos suelen estar ocultos, ser invisibles o inconscientes. ${ }^{46}$ Las normas y valores cambian con el tiempo. En lugar de aceptar como legítima una acción desinteresada cualquiera, utilizo los acuerdos de la Organización de las Naciones Unidas como punto de referencia para el análisis empírico que se presenta a continuación. ${ }^{47}$

${ }^{43}$ Cf. Dieter y Kumar, art. cit., y Chantal Nadeau, “вв and the beasts: Brigitte Bardot and the Canadian seal controversy", Screen, vol. 37, núm. 3, 1996, pp. 240-250.

${ }^{44}$ B. Schmitz y V. Then, "Legitimation durch Narration - Bindungskräfte durch das Erzählen von Geschichten” en Helga Hackenberg y Stefan Empter, Social Entrepreneurship - Social Business: Für die Gesellschaft unternehmen, Wiesbaden, VS Verlag für Sozialwissenschaften, 2011.

${ }^{45}$ C. Offe, "Wessen Wohl ist das Gemeinwohl?" en Harald Bluhm y Herfried Münkler, Gemeinwohl und Gemeinsinn: Rhetoriken und Perspektiven sozial-moralischer Orientierung, Berlín, Akademie Verlag, 2002.

46 Partzsch, art. cit.

${ }^{47}$ L. Partzsch, Global Governance in Partnerschaft: Die EU-Initiative "Water for Life”, Baden-Baden, Nomos, 2007, pp. 101-102. 
En otras palabras, el consenso de la ONU, que requiere la aprobación de todos los Estados-nación, funge como estructura para definir los objetivos universales y el bien común en el mundo. Al analizar la legitimidad del poder de las celebridades por su influencia política, se puede verificar que la acción política cumpla con los acuerdos de la onU y, al mismo tiempo, cuestionar si hay conflictos políticos relacionados con sus demandas. De todas maneras, según la teoría democrática, la "legitimación por medio de la influencia política" sólo puede fungir como una lenitiva e indirecta forma de legitimidad, ${ }^{48}$ que requiere, para cumplirse, de una amplia participación del demos (segundo criterio) y del control y la rendición de cuentas (tercer criterio).

\section{Legitimidad por amplia participación del demos}

En un caso ideal de toma legítima de decisiones, las de la ONU y otros acuerdos internacionales son el resultado de procedimientos justos con una participación amplia del demos. ${ }^{49} \mathrm{El}$ demos representa el total de personas afectadas y ciudadanos. La Asamblea General de las Naciones Unidas, cuyos miembros tienen la misma representación, es lo que más se acerca a representar el demos mundial en el ámbito internacional. En este modelo, los delegados hablan por sus ciudadanos trasnacionalmente. Este esquema implica una serie de déficits democráticos que incluye largas cadenas de legitimación entre quienes toman las decisiones y los ciudadanos particulares. Aún más, los gobiernos, que operan como órganos ejecutivos en el terreno nacional, toman decisiones legislativas en el internacional. Además, en la Asamblea General todos los países cuentan con los mismos derechos de voto independientemente del tamaño de sus poblaciones. En su carácter de miembros permanentes del Consejo de Seguri-

${ }^{48}$ Scharpf, op. cit., p. 349.

${ }^{49}$ Partzsch, op. cit., pp. 102-103. 
dad, China, Estados Unidos, Francia, el Reino Unido y Rusia tienen derechos especiales. A fin de cuentas, tanto en la Asamblea General, como en el Consejo de Seguridad, hay varios países miembros no democráticos, cuyos derechos de veto pueden resultar cruciales. ${ }^{50}$

En estas circunstancias, muchos académicos han abrazado el surgimiento de una sociedad trasnacional de ciudadanos, en la cual éstos se organicen a sí mismos en lugar de dejar todas las decisiones en manos de los delegados estatales. ${ }^{51}$ Tanto en la Conferencia de las Naciones Unidas sobre el Medio Ambiente y el Desarrollo -la llamada "Cumbre para la Tierra", que aprobó la Convención Marco de las Naciones Unidas sobre el Cambio Climático en 1992-, como la subsiguiente Conferencia de las Naciones Unidas sobre el Desarrollo Sostenible de 2012, se puso la atención en la importancia de que hubiera una participación de la sociedad civil en favor del desarrollo sostenible. Sin embargo, la inclusión de actores no estatales y ciudadanos individuales en las relaciones internacionales, raramente significa que su desempeño sea exclusivamente en nombre propio. Es particularmente común que las celebridades tomen cartas en el asunto por un tercero, lo que conduce a una situación en la que, por un lado, la participación de estas figuras públicas aumenta la percepción de que las decisiones son legítimas (porque hay "ciudadanos" de renombre involucrados y supuestamente una mayor participación del demos) y, por otro, sólo en los casos en que todos los intereses afectados y las perspectivas están realmente representados de manera equilibrada, se puede considerar que la inclusión de los actores involucrados promueve la democracia según el statu quo. ${ }^{52}$

${ }^{50}$ Cf. J. Nye, “Globalization's democratic deficit: How to make international institutions more accountable”, Foreign Affairs, vol. 80, núm. 2, 2001, pp. 2-6; y Wolf, art. cit.

51 J. Steffek, C. Kissling y P. Nanz (eds.), Civil society participation in European and global governance: a cure for the democratic deficit?, Basingstoke, Palgrave MacMillan, 2008.

52 Cf. Renate Mayntz, Legitimacy and compliance in transnational governance, Köln, Max-Planck-Institut für Gesellschaftsforschung, 2010, en www. 
¿Qué significa esto en lo tocante a la legitimidad del poder de las celebridades en las Relaciones Internacionales? Se puede evaluar la legitimidad de las celebridades por una amplia participación del demos según la contribución que estas figuras públicas hagan para que haya una representación más equilibrada de todos los intereses y perspectivas en el proceso de toma de decisiones y para procurar que las decisiones se basen en consensos. Dar voz a posturas no dominantes no implica tomar una dirección opuesta a coadyuvar en la implementación de los acuerdos de la ONU (primer criterio), si se acepta que estas metas son el resultado de procesos anteriores de construcción de consensos (en que los objetivos pueden estar bajo constante escrutinio y sujetos a las dinámicas políticas). En este sentido, los individuos con una limitada legitimidad por participación amplia del demos (segundo criterio) pueden influir legítimamente en el proceso de toma de decisiones, si respaldan el cumplimiento de decisiones que surgieron de procedimientos anteriores justos (primer criterio). Además, en un caso ideal de legitimidad también debería haber procedimientos que permitiesen que las personas afectadas demandaran la revisión o revocación de cualquier decisión política (tercer criterio).

\section{Legitimidad por control y rendición de cuentas}

Entre más exclusivas sean las estructuras de toma de decisiones, más relevantes se vuelven los mecanismos de rendición de cuentas entre "los de adentro", que deciden, y "los de afuera", que están sujetos a lo que decidan aquéllos. ${ }^{53}$ Mientras que, cuando menos en los Estados democráticos, la población elige gobiernos que representan sus intereses y actúan en su representación (relación de poder bidireccio-

mpifg.de/pu/workpap/wp10-5.pdf, consultado el 2.VIII.2012; y Partzsch, op. cit., pp. 102-103.

53 Partzsch, op. cit., p. 105. 
nal), en la onU y otras organizaciones internacionales, los actores no estatales, como la mayoría de las celebridades, no surgen de las urnas. ${ }^{54}$ Por tanto, si la gente no está satisfecha con su influencia, no pueden ni responsabilizarlos políticamente ni retirarles el voto. Por consecuencia, diferentes autores sostienen que, además de la legitimidad por influencia política (primer criterio) y aquella por la amplia participación del demos (segundo criterio), se requiere también de una legitimidad orientada al rendimiento por el control y la rendición de cuentas (tercer criterio). ${ }^{55}$ Del siguiente modo, Hesselmann define las relaciones de poder bidireccional de esta índole:

La rendición de cuentas establece una relación formal entre dos series de actores o instituciones, en la cual una de las partes ("la que rinde cuentas") está obligada a dar a conocer, explicar y justificar sus acciones a la otra ("a la que se rinde cuentas") y afronta las consecuencias en caso de no haber cumplido ni con los estándares ni las expectativas. ${ }^{56}$

Hesselmann sugiere un modelo de tres fases para rendir cuentas (véase cuadro 2). En la primera, la más básica de todas, a quien se rinda cuentas (por ejemplo, las personas afectadas o, en dado caso, las instituciones del sistema de la ONU que involucran a celebridades en sus campañas) tiene el derecho de exigir transparencia e información de quienes están sujetos a rendir cuentas (por ejemplo, las celebridades que actúan políticamente). La procuración, entendida como

${ }^{54}$ Gebauer, art. cit., pp. 103-104.

55 Cf. R. O. Keohane y J. S. Nye, "Redefining accountability for global governance”, en M. Kahler y D. A. Lake (eds.), Governance in a global economy: Political authority in transition, Oxford, Princeton University Press, 2003; y Take, art. cit.

56 "The limits of control: The accountability of foundations and partnerships in global health" en S. Rushton y O. D. Williams (eds.), Partnerships and foundations in global health governance, Basingstoke, Palgrave MacMillan, 2011, p. 229. 
la consecución de un resultado buscado, es lo que define la segunda fase: la capacidad de sancionar o premiar una conducta. La tercera se alcanza si quien está sujeto a rendir cuentas reacciona a la retroalimentación de quien las recibe y cambia su comportamiento. ${ }^{57}$ En su conjunto, las tres fases se refieren al tercer criterio de legitimidad, según se ha descrito en este artículo.

Los círculos de retroalimentación informal no se han considerado en este modelo. Aunque por sí mismos pueden ser menos democráticos que los mecanismos formales de rendición de cuentas, el control y las sanciones del comportamiento de los actores no estatales se pueden aplicar únicamente cuando se basan en autoevaluaciones voluntarias y en los compromisos autoimpuestos correspondientes. Además, los actores no estatales suelen utilizar mecanismos de rendición de cuentas de índole social para responsabilizar, en nombre de las personas afectadas, a los gobiernos y a las organizaciones internacionales de sus omisiones. ${ }^{58}$

Si se aplicaran, los mecanismos voluntarios de rendición de cuentas y control coadyuvarían a la democracia. Propongo modificar, por tanto, el modelo de Hesselmann y complementar sus criterios de una más inflexible rendición de cuentas en el ámbito político con los de una más indulgente por lo que hace a la sociedad (véase cuadro 2): en la primera fase, quienes están sujetos a rendir cuentas (por ejemplo, las celebridades) voluntariamente pueden también permitir mayor transparencia y ofrecer información sobre sus acciones a las personas afectadas y al público en general. El control social es lo que define la segunda fase modificada. Por ejemplo, las personas afectadas y los actores que hablan en su representación pueden "señalar y denunciar" a quien debe rendir cuentas y expresar su aprobación, participando en ciertas actividades políticas, o su oposición, absteniéndose de ellas. En la tercera fase, quienes rinden cuentas cambian más o menos

${ }^{57}$ Ibid., pp. 233-235.

${ }^{58}$ Dieter y Kumar, art. cit. 
voluntariamente su comportamiento como reacción a la crítica o como resultado del aprendizaje (por ejemplo, el conocimiento nuevo de las condiciones locales). En un análisis empírico, podemos evaluar caso por caso para saber si el que rinde cuentas cambia su comportamiento y si tal cambio corresponde al reclamo o la opinión de aquel a quien se rinde cuentas.

¿Esto qué significa para la legitimidad del poder de las celebridades? Las celebridades pueden incrementar su legitimidad por el control y la rendición de cuentas más allá del Estado-nación, si dan voz a personas que, de otro modo, no serían escuchadas, y si, en nombre de las personas afectadas, responsabilizan a los gobiernos y las organizaciones internacionales del compromiso (o la falta de éste) con acuerdos anteriores. ${ }^{59}$ Sin embargo, si las celebridades determinan las acciones e incluso las ideas de otros, deberían rendir cuentas al demos (o a las instituciones que lo representan, como la Asamblea General de las Naciones Unidas) afectado por sus acciones. Con el propósito de evaluar la legitimidad de las celebridades en el ámbito de las Relaciones Internacionales, debe preguntarse, en cada caso, cuántos mecanismos tanto formales como informales de control y rendición de cuentas hay para el poder de las celebridades.

\section{LA LEGITIMIDAD DEL PODER DE LAS CELEBRIDADES}

Las celebridades ejercen su poder en el ámbito de las Relaciones Internacionales. ${ }^{60}$ En las siguientes secciones se analizará la legitimidad de dos celebridades seleccionadas: Leonardo DiCaprio y Daryl Hannah. Con base en este examen, se estudiará si enriquecen o depauperan la legitimidad de los procesos de toma de decisiones que trascienden el ámbito del Estado-nación. El cuadro 1 ofrece una perspectiva general

${ }^{59} I d$.

60 Partzsch, art. cit. 
de los tres criterios y las preguntas utilizadas para codificar el conjunto de datos empíricos.

\section{Influencia política: el establecimiento (no) controvertido de la agenda}

El primer criterio sobre legitimidad es la influencia política. Según este criterio, el poder de las celebridades sólo se puede considerar legítimo cuando se ejerce para implementar objetivos universales, que aquí se definen como aquellos sobre los cuales hay un acuerdo previo de las Naciones Unidas. Según se describió anteriormente, DiCaprio y Hannah fungen como catalizadores para lograr que ciertos temas medioambientales se incluyan en la agenda política global, lo que, desde la Declaración de Río de 1992, es un objetivo universal. Ambas celebridades son modelos de conducta. Aunque hayan enormes controversias sobre el cambio climático en los Estados Unidos, durante la cop 21, celebrada en París en 2015, la Organización de las Naciones Unidas acordó un plan de acción global que marca el límite del calentamiento global por debajo de los $2^{\circ} \mathrm{C}$. El acuerdo entrará en vigor en 2020.

Cuando tomó la palabra en el marco de la Cumbre sobre el Clima de la onu, celebrada en Nueva York, en septiembre de 2014, DiCaprio instó a los delegados a actuar: "Y ahora el turno es SUYO; el momento para enfrentar el mayor reto de nuestra existencia en el planeta es este momento". ${ }^{61}$ Las declaraciones políticas sobre el cambio climático en su discurso de aceptación del Oscar en 2016 fueron igualmente vagas. Dos meses después de que la comunidad internacional acordara en París el límite de menos de $2{ }^{\circ} \mathrm{C}$, afirmó: "El cambio climático es una verdad y ocurre en este instante... No echemos el planeta en saco roto". ${ }^{62}$ Por el contrario, ésta

61 "Leonardo DiCaprio at the Un...".

${ }^{62}$ DiCaprio, "2016 Oscar acceptance speech for best actor", 2016, en www.oscar.go.com/news/winners. 
es la exigencia más precisa que haya pronunciado como previsión política: "tasar las emisiones de carbono y eliminar los subsidios gubernamentales para las compañías que usan petróleo, gas y carbón". ${ }^{63}$ Aunque los mercados del carbón se han vuelto sumamente controvertidos, ${ }^{64}$ las exigencias de DiCaprio concuerdan en general con las de la ONU. ${ }^{65}$

Su postura, sin embargo, difiere de las protestas de Hannah en contra del oleoducto Keystone XL en Estados Unidos y Canadá. Hannah ha sido arrestada repetidamente por "desobediencia civil". Hay que recordar cuando en 2006 se encaramó a un nogal junto a otros activistas en un intento por salvar un rancho urbano en una zona deprimida de Los Ángeles, después de que un juez emitiera una orden de desalojo (el terreno privado había sido limpiado para construir una bodega). En 2011, para protestar en contra de tal oleoducto, Hannah montó una campaña de dos semanas en la Casa Blanca y fue arrestada junto a otras casi mil personas, entre las que se encontraban la actriz canadiense Margot Kidder y la activista de la misma nacionalidad Naomi Klein. ${ }^{66}$

Las exigencias de Hannah para el futuro abastecimiento de combustible claramente superaban y parcialmente contradecían los objetivos de los Estados Unidos y de las Naciones Unidas a este propósito. Mientras que el programa de estándares de combustible renovable de los Estados Unidos (RFS, por sus siglas en inglés) se centra en la reducción de emisiones de carbono y la autonomía estadounidense con respecto de la importación de petróleo, Hannah fundó la Alianza para un Biodiesel Sostenible a fin de promover una producción de combustibles renovables en la Unión Ameri-

63 "Leonardo DiCaprio at the Un...".

64 McCauley, art. cit.

65 O. Chukwumerije, H. Bulkeley y H. Schroeder, "Conceptualizing climate governance beyond international regime", Global Environmental Politics, vol. 9, núm. 1, 2009, pp. 58-78.

66 "Keystone XL Pipeline key events...". 
cana de carácter local y basada en la comunidad. ${ }^{67}$ De hecho, en un video que puede verse por Internet, Hannah bebe el aceite vegetal que usa como combustible para demostrar cuán saludables y seguros son los combustibles renovables. ${ }^{68}$

Hay un acalorado debate sobre la influencia de la producción de biocombustibles en el suministro de alimentos, el costo de los productos alimentarios y la escasez de éstos, en particular en el Sur global. ${ }^{69}$ La Organización de las Naciones Unidas ha advertido acerca de los peligros ambientales y de seguridad alimentaria en el Sur global como consecuencia de haber desarrollado masivamente biocombustibles en los Estados Unidos. Jean Ziegler, relator especial de la onU para el Derecho a la Alimentación, dijo que los biocombustibles son "crimen de lesa humanidad". En 2008, cuando se dispararon drásticamente los precios de los alimentos en el Sur global, exigió que se prohibiera la producción de biocombustibles en el mundo entero durante cinco años. ${ }^{70}$ Paradójicamente, Hannah bebe biodiesel para promoverlo, desatendiendo por completo la controversia sobre alimentación y combustible hasta el punto de poder contravenir su propósito original. ${ }^{71}$ Por el contrario, al beber biodiesel para promoverlo, Hannah desestima por completo la controversia del asunto, a la vez que potencialmente la replantea. Por tanto, su compromiso político resulta aún más controvertido que la vaga campaña de DiCaprio para salvar el planeta. Comparada con éste, por tanto, no podrá cumplir con ninguno de sus propósitos sin la legitimidad que confiere la influencia política.

67 "Sustainable Biodiesel Alliance. Homepage", SBA, en www.sustainablebiodieselalliance.com.

68 "Daryl Hannah does Bio Diesel!", Sunking67, 2016, en www.youtube.com/watch?v=pUZUfRJD0aQ, consultado el 20.VII.2016.

${ }^{69}$ Sengers, Raven y Van Venrooij, art. cit.

${ }^{70} \mathrm{~J}$. Ziegler, Report of the Special Rapporteur on the right to food: GE.0810098, 2008, en www.humanrights.ch/home/upload/pdf/080311_bericht_ziegler.pdf, consultado el 3.III.2008.

71 "Daryl Hannah does Bio...". 
En suma, aunque se pueda simpatizar con la postura más radical de Hannah, con base únicamente en el primero de los criterios, el de la legitimidad por influencia política, el compromiso de DiCaprio debe considerarse más legítimo que las acciones de Hannah. La onu acepta la ayuda que DiCaprio brinda para que el cambio climático figure como uno de los temas en la agenda pública, a condición de que no vaya más allá de las exigencias propias de la Organización. Usa de su poder para que puedan alcanzarse las metas comunes a todos, mientras que Hannah usa de su fama para promover posturas de activistas norteamericanos que no gozan de consenso.

\section{Voceros de ecologistas norteamericanos}

El segundo criterio corresponde a la inclusión equilibrada de intereses en el ámbito de las relaciones internacionales, particularmente los de la sociedad civil. Los de DiCaprio y Hannah suelen presentarse como casos de defensa en nombre de movimientos ambientales. En el discurso de aceptación por su nombramiento como embajador de buena voluntad de las Naciones Unidas y, por tanto, con la autorización de esta organización internacional, Di Carpio afirmó que hablaba en nombre de "las cuatrocientas mil personas que habían marchado por las calles de Nueva York... y los miles de millones más en todo el mundo que quieren resolver nuestra crisis climática". ${ }^{72}$ En Internet podemos encontrar muchas fotografías de DiCaprio y Hannah entre los manifestantes. Hannah estuvo en el grupo que, así como suena, se subió a un árbol para intentar detener la demolición de un huerto comunitario en Los Ángeles. ${ }^{73}$ "Tengo plena confianza en que

72 "Leonardo DiCaprio at the UN...".

73 Cf. P. Harris, "Hollywood stars rally to save farm", The Guardian Observer, 2006, en www.theguardian.com; y R. Prince, "Leonardo DiCaprio and Sting among thousands on New York climate change march", The Telegraph, 2016, en www.telegraph.co.uk, consultado el 10.I.2016. 
estoy haciendo lo moralmente correcto: asumir una postura solidaria con los granjeros", explicó a los medios de comunicación en relación con su papel en la protesta. ${ }^{74}$

Mientras que en casos populares, como el de la campaña en contra de las minas de tierra que (con el apoyo de la princesa Diana y Bono, entre otros) dio origen a la Convención sobre la Prohibición de Minas de Tierra en 1997, las celebridades claramente no estaban facultadas por el movimiento en su conjunto, ${ }^{75}$ hay evidencia en los medios de comunicación de que, por lo menos DiCaprio, estaba entre las personas que marcharon por las calles de Nueva York y de que Hannah se encontraba entre los participantes de distintas actividades de esa índole. ${ }^{76}$ Como el público en general no suele estar al tanto de las polémicas internas de los movimientos ecologistas, la percepción es que las celebridades representan la escena completa y, por tanto, se considera que aportan perspectivas adicionales, y con frecuencia más radicales, que dan equilibrio a las posturas gubernamentales y empresariales en temas ambientales en el marco de las Relaciones Internacionales. Sin embargo, si evaluamos el contenido de los mensajes de DiCaprio y Hannah, encontraremos que no necesariamente han contribuido con contenido adicional a los debates políticos por lo que hace al medio ambiente. Su repetición de posturas dominantes (tasar el precio del carbón, promover los biocombustibles, etc.) puede incluso haber fortalecido las asimetrías de poder al mantener en la sombra las posturas marginalizadas y de mayor alcance (y más controvertidas). Este efecto debilita los procedimientos democráticos. Así, por un lado, se considera que las celebridades apoyan una participación más amplia del demos y, por otro, DiCaprio y Hannah únicamente transmiten las posturas "dominantes" de los grupos de activistas

74 "Daryl Hannah arrested after garden protest", The Associated Press, 2016, en www.today.com/id/13297274\#.VrJhMUbGgwI, consultado el 10.I.2016.

${ }^{75}$ Cf. Gebauer, art. cit., pp. 103-104, y Partzsch, art. cit.

${ }^{76}$ Harris, art. cit. 
norteamericanos, cuya voz ya se ha escuchado con anterioridad en el campo de las Relaciones Internacionales. Por tanto, su legitimidad democrática, según una participación más amplia del demos en las Relaciones Internacionales, resulta ambivalente.

La información voluntaria en vez de la rendición de cuentas en el ámbito político

Entre más se cuestione qué actores deben participar en el proceso de toma de decisiones (segundo criterio), más relevantes serán los mecanismos de rendición de cuentas entre "los de adentro", que toman las decisiones, y "los de afuera", a quienes afectan esas decisiones. ${ }^{77} \mathrm{Su}$ popularidad permite a las celebridades penetrar círculos exclusivos más fácilmente y hablar en nombre de los que están fuera. Al hacer públicos los problemas medioambientales, tanto DiCaprio como Hannah contribuyen a responsabilizar a los gobiernos. Sin embargo, si las celebridades afirman actuar en representación de quienes están por fuera, surge la pregunta de si estos últimos pueden controlar a las primeras y si éstas, a su vez, les rinden cuentas (relación bilateral de poder). Hay mecanismos sociales e informales entre las celebridades y los grupos ecologistas que se suman a los procedimientos de control y rendición de cuentas. Partiendo de la base de que la legitimidad y la democracia en el ámbito de las Relaciones Internacionales son un continnum, utilizaremos como referencia el modelo trifásico de Hesselmann (modificado) para analizar si DiCaprio y Hannah cumplen con los criterios tanto de una más rigurosa rendición de cuentas en el ámbito político como de una rendición de cuentas lenitiva en el social (véase cuadro 2 ).

77 Partzsch, op. cit., pp. 104-105. 


\section{Cuadro 2}

Rendición de cuentas o rigurosa o lenitiva

(tercer criterio de legitimidad)

\begin{tabular}{|c|c|c|}
\hline $\begin{array}{l}\text { Modelo trifásico de } \\
\text { rendición de cuentas }\end{array}$ & $\begin{array}{l}\text { Rendición de cuentas rigurosa en } \\
\text { el ámbito político }{ }^{\mathrm{a}}\end{array}$ & $\begin{array}{c}\text { Rendición de cuentas lenitiva en el } \\
\text { ámbito social }\end{array}$ \\
\hline Primera fase & $\begin{array}{l}\text { A quien se rinde cuentas (por } \\
\text { ejemplo, las personas afecta- } \\
\text { das) tiene derecho de exigir } \\
\text { transparencia e información de } \\
\text { quienes deben rendirlas (por } \\
\text { ejemplo, las celebridades). }\end{array}$ & $\begin{array}{l}\text { Transparencia por informar } \\
\text { voluntariamente. }\end{array}$ \\
\hline Segunda fase & $\begin{array}{l}\text { A quien se rinden cuentas pue- } \\
\text { de emplear mecanismos de } \\
\text { procuración para sancionar y } \\
\text { premiar conductas. }\end{array}$ & $\begin{array}{l}\text { Control social (por ejemplo, "se- } \\
\text { ñalar y denunciar", participar o } \\
\text { no participar). }\end{array}$ \\
\hline Tercera fase & $\begin{array}{l}\text { Quienes rinden cuentas adap- } \\
\text { tan su comportamiento al de } \\
\text { quien demanda la rendición } \\
\text { de cuentas. }\end{array}$ & $\begin{array}{l}\text { Cambio de comportamiento se- } \\
\text { gún las críticas o como resultado } \\
\text { del aprendizaje (por ejemplo, el } \\
\text { conocimiento nuevo sobre con- } \\
\text { diciones locales). }\end{array}$ \\
\hline
\end{tabular}

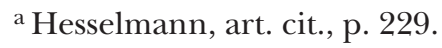

Cuando DiCaprio habló en nombre de "los mil millones de personas que en el mundo quieren remediar nuestra crisis climática"78 y Hannah afirmó actuar "solidariamente con los granjeros"79, a la luz de la teoría sobre la democracia, estas celebridades se hicieron con la responsabilidad de pedir cuentas en nombre de esos manifestantes y granjeros. Sin embargo, no hay mecanismo formal para trasladar la rendición de cuentas en el ámbito político. Por tanto, si seguimos el modelo original de Hesselmann sobre este tipo de rendición de cuentas, las celebridades no alcanzan siquiera la primera fase. Los manifestantes ecologistas no tienen ningún

78 "Leonardo DiCaprio at the UN...".

79 "Daryl Hannah arrested...". 
derecho formal de exigir transparencia e información (primera fase; véase cuadro 2). Como parte de la campaña en contra de las minas de tierra, en febrero de 1998, una asamblea general acordó procedimientos para el proceso interno de toma de decisiones y de representación. Pero para ese momento, celebridades como la princesa Diana y Bono ya habían popularizado algunas demandas (parciales) entre un público más amplio. ${ }^{80}$ En la mayoría de los casos, las redes transnacionales no cuentan con ese tipo de procedimientos formales. Con todo, hay evidencia en los medios de comunicación de que comúnmente DiCaprio y Hannah, según se ha mencionado, están con la gente y protestan a su lado. Ante esta interacción social, podrían funcionar otros mecanismos más complejos en lo que atañe a la rendición lenitiva de cuentas.

La gente se puede acercar a DiCaprio y Hannah cuando están manifestándose al lado de otros activistas. Además, ambas celebridades han explicado en entrevistas por qué decidieron apoyar ciertos proyectos y campañas. Si bien las personas afectadas dependen de información otorgada de manera voluntaria, cuando las celebridades la proporcionan cumplen con la primera fase de la rendición de cuentas en el ámbito social. Un debate público más crítico sobre el poder de las celebridades también podría contribuir a la rendición de cuentas, por ejemplo, señalando y denunciando. Si DiCaprio y Hannah instan a comprar o boicotear determinados productos (por ejemplo, biocombustible), los consumidores pueden participar o no, lo cual define la segunda fase de la rendición lenitiva de cuentas en el ámbito social. En la tercera y última fase, las celebridades podrían cambiar su comportamiento según las críticas o como resultado del aprendizaje. Es posible que, por causa de las críticas a la mercantilización de la naturaleza, DiCaprio no vuelva a reclamar sobre tasar el precio del carbón (y permanezca en la indefinición para mantenerse en la línea de la ONU). Sin embargo, no hay evidencia alguna de que a quienes se rinde cuentas

${ }^{80}$ Gebauer, art. cit., pp. 103-104. 
hayan ejercido algún tipo de control sobre DiCaprio y Hannah o los hayan obligado a cambiar sus respectivas posturas. Ambas celebridades mantuvieron su posición política a lo largo del periodo de evaluación de este estudio. Por tanto, aunque puedan aumentar la legitimidad del control y la rendición de cuentas más allá del Estado-nación, responsabilizando de manera social a los gobiernos y las empresas privadas del (no) cumplimiento de acuerdos previos, las celebridades no satisfacen el tercer criterio, puesto que no rinden cuentas sino a ellas mismas.

\section{Conclusiones}

¿El poder que tienen las celebridades en el ámbito de las Relaciones Internacionales es legítimo? ¿El creciente compromiso de las celebridades con distintas causas internacionales enriquece o depaupera el proceso de toma de decisiones que trasciende el Estado-nación? Como se ha observado, las demandas políticas de DiCaprio, que han suscitado controversia, están en concordancia con el consenso de las Naciones Unidas. En cambio, la postura política de Hannah no cuenta, en términos generales, con el apoyo de la comunidad internacional. Las posiciones de ambas celebridades son cuestionadas al interior de los movimientos ecologistas globales, en particular las relativas a los biocombustibles y a la tasa de los precios del carbón. El propósito de este artículo es contribuir a un debate más matizado sobre la legitimidad de las celebridades en el campo de las Relaciones Internacionales. Inicialmente formulé tres criterios de legitimidad que después se utilizaron para explorar de forma ilustrativa la legitimidad del poder de las celebridades en torno a DiCaprio y Hannah. Primero, la legitimidad por influencia política; segundo, la legitimidad por amplia participación del demos, tercero, la legitimidad por un modelo trifásico de rendición de cuentas. Como conclusión, ahora se esboza la ambivalencia del poder de las celebridades según su legitimidad -de nuevo, con base 
en estos tres criterios. El supuesto subyacente es que, al cumplir con estos criterios, las celebridades incrementan su legitimidad y contribuyen a la democracia más allá del Estado-nación. Por el contrario, si no cumplen con estos criterios, su creciente compromiso incluso fortalece los déficits democráticos en el orden internacional.

En primer lugar, las personas suelen tener el poder político -incluido el de las celebridades- por legítimo, cuando sirve de manera eficaz al bien común. Mientras sus demandas apoyen objetivos universales y no contradigan ninguna postura sobre la cual haya un acuerdo de la comunidad internacional, las celebridades pueden elaborar a partir de una legitimidad por influencia política. A la luz de este primer criterio, colocar los temas medioambientales en la agenda pública legitima a las celebridades. La exigencia de DiCaprio de tomar el cambio climático en serio contribuye a los esfuerzos de las Naciones Unidas de poner un límite de $2^{\circ} \mathrm{C}$ al calentamiento global. En contraste, Hannah suele adoptar posturas más polémicas. Por ejemplo, su posicionamiento sobre los biocombustibles ignora totalmente el dilema internacional "comida contra combustible". Por tanto, aunque las vagas exigencias de DiCaprio puedan tener menor influencia y posiblemente sirvan a la protección del medio ambiente y a la sostenibilidad con menor vigor que las de Hannah, según el primer criterio, el poder del actor debe considerarse legítimo, mientras que el de la actriz no puede cimentarse en una legitimidad por influencia política.

En segundo lugar, los expertos consideran que la amplia participación del demos promueve la legitimidad y que la participación de los representantes de la sociedad civil puede pesar más que los déficits democráticos en la política intergubernamental. Como ha quedado demostrado, las celebridades tienen la oportunidad de incluir temas medioambientales adicionales en la agenda internacional, lo que a su vez podría conducir a una representación mayor de inquietudes en el campo de las Relaciones Internacionales. Sin embargo, en los hechos, hemos observado que los estudios de caso 
indican que las celebridades únicamente comunican las posturas de los grupos de activistas dominantes de Norteamérica y no toman en cuenta demandas marginadas y de mayor alcance, que, con frecuencia, quedan ocultas tras el poder de las celebridades. Este efecto depaupera los procedimientos democráticos. Muchos activistas del Sur global y de otros lugares rechazan los biocombustibles y tasar el precio del carbón. Por tanto, en lo que atañe a una participación amplia del demos, la legitimidad democrática de DiCaprio y Hannah es ambivalente.

En tercer lugar, las decisiones y acciones políticas pueden considerarse mayormente legítimas, si quienes las toman y ejercen a nombre de otros están bajo el control de estos últimos, a quienes también los primeros rinden cuentas. Las celebridades responsabilizan, junto con los grupos de activistas, a los gobiernos, a pesar de que ellas mismas no asuman formalmente sus responsabilidades ante nadie. De la participación de DiCaprio y Hannah en actos de protesta se podrían desprender mecanismos informales de retroalimentación y control, pero este estudio no ha encontrado que, durante su periodo de evaluación, esta interacción social haya conducido a que las celebridades reaccionaran y se retractaran de una postura específica.

Por medio de su compromiso político en el ámbito de las Relaciones Internacionales, las celebridades ponen sobre la mesa de la agenda pública problemas que inquietan a la ciudadanía. Los medios de comunicación presentan a DiCaprio y Hannah como voceros de los movimientos ecologistas y el público en general los recibe como actores legítimos en el campo de las Relaciones Internacionales. Si consideramos su compromiso con las "buenas causas", los cuestionamientos sobre su legitimidad parecen fuera de lugar, en especial porque frecuentemente su influencia termina por establecer una agenda no controvertida. Sin embargo, mientras no se tomen en cuenta los conflictos en relación con las alternativas a sus agendas medioambientales, la legitimidad del poder de las celebridades seguirá siendo ambivalente. Aunque 
el compromiso de Hannah -entre otras celebridades- en contra del Keystone XL pueda haber influido a favor de la negativa del gobierno del presidente Obama sobre el tema-y a pesar de que la promoción que hace la actriz de los biocombustibles pueda resultar igualmente eficaz-, su participación no necesariamente coadyuva a una democracia que trascienda el Estado-nación. Con todo, el nuevo auge de poder de las celebridades en las Relaciones Internacionales podría depauperar aún más la democracia y poner en desventaja aún mayor a actores y posturas ya de suyo marginados.

\section{Traducción de Gonzalo Celorio Morayta}

\section{BibLIOGRAFÍA}

Andersen, Allison, "«Together we can save the Arctic»: Celebrity advocacy and the Rio Earth Summit 2012", Celebrity Studies, vol. 3, núm. 4, 2013, pp. 339-352, doi:10.1080/19392397.2013.831617.

Brunnengräber, Achim, Klein Ansgar y Heike Walk (eds.), NGos als Legitimationsressource: Zivilgesellschaftliche Partizipationsformen im Globalisierungsprozess, Opladen, Leske \& Budrich, 2001.

Clifford, Bob, The Marketing of rebellion: Insurgents, media, and international activism, Cambridge, University Press, 2005.

Chouliaraki, Lilie, "The theatricality of humanitarianism: A critique of celebrity advocacy", Communication and Critical/Cultural Studies, vol. 9, núm. 1, 2011, pp. 1-21.

Cooper, Andrew F., Celebrity Diplomacy, Colorado, Paradigm, 2008.

"Daryl Hannah arrested after garden protest", The Associated Press, 2016, en www.today.com/id/13297274\#.VrJhMUbGgwI

"Daryl Hannah. Charity Work, Events and Causes", en www.looktothestars.org/celebrity/daryl-hannah

"Daryl Hannah does Bio Diesel!", Sunking67, 2016, en www.youtube.com/watch?v=pUZUfRJD0aQ

DiCaprio, Leonardo, "2016 Oscar acceptance speech for best actor", 2016, en www.oscar.go.com/news/winners

Dieter, Heribert y Rajiv Kumar, "The downside of celebrity diplo- 
macy: The neglected complexity of Development", Global Governance, vol. 14, núm. 3, 2008, pp. 259-264.

Fuchs, Doris, "Commanding Heights? The Strength and Fragility of Business Power in Global Politics", Millennium: Journal of International Studies, vol. 33, núm. 3, 2005, pp. 771-801.

Gebauer, Thomas, “«...von niemandem gewählt!»: Über die demokratische Legitimation von NGO", en Ulrich Brand, Alex Demirovic, Chistoph Görg y Joachim Hirsch (eds.), Nichtregierungsorganisationen in der Transformation des Staates, Münster, Westfälisches Dampfboot, 2001.

Hannah, Daryl, "Homepage DH Love Life", 2016, en www.dhlovelife.com

Harris, Paul, "Hollywood stars rally to save farm", The Guardian Observer, 2006, en www.theguardian.com

Hesselmann, Elena, "The limits of control: The accountability of foundations and partnerships in global health" en Simon Rushton y Owain D. Williams (eds.), Partnerships and foundations in global health governance, Basingstoke, Palgrave MacMillan, 2011, pp. 228-252.

"Jennifer Lopez presents UNiCEF Women of Compassion Award", 30 de noviembre de 2015, en www.looktothestars.org/news

Kaldor, Mary, "The idea of global civil society", International Affairs, vol. 79, núm. 3, 2003, pp. 583-593.

Keohane, Robert O. y Joseph S. Nye, "Redefining accountability for global governance”, en Miles Kahler y David A. Lake (eds.), Governance in a global economy: Political authority in transition, Oxford, Princeton University Press, 2003.

"Keystone XL Pipeline key events from proposal to Obama's rejection”, Huffington Post, 2016, en www.huffingtonpost.ca

"Leonardo DiCaprio. Charity Work, Events and Causes", 10 de julio de 2017, en www.looktothestars.org

"Leonardo DiCaprio at the un: «Climate change is not hysteria -it's a fact»", 2015, The Guardian, en www.theguardian.com

Mayntz, Renate, Legitimacy and compliance in transnational governan$c e$, Köln, Max-Planck-Institut für Gesellschaftsforschung, 2010, en www.mpifg.de/pu/workpap/wp10-5.pdf 
McCauley, Douglas J., "Selling out nature: Commentary", Nature Climate Change, vol. 443, núm. 7107, 2006, pp. 27-28, doi:10.1038 / 443027a.

Macdonald, Laura, "Globalizing civil society: Interpreting international NGOs in Central America”, Millennium: Journal of International Studies, vol. 23, núm. 2, 1994, pp. 267-285.

Nadeau, Chantal, "вв and the beasts: Brigitte Bardot and the Canadian seal controversy", Screen, vol. 37, núm. 3, 1996, pp. 240-250. NASA, "Global Climate Change", 2017, en www.climate.nasa.gov

Neyer, Jürgen, "Die Stärke deliberativer politischer Theorien und das Elend der orthodoxen Demokratietheorie: Eine Replik auf Marcus Höreth", Zeitschrift für Politikwissenschaft, vol. 19, núm. 3, 2009, pp. 331-358.

Nullmeier, Frank, Anna Geis y Christopher Daase, "Der Aufstieg der Legitimitätspolitik: Rechtfertigung und Kritik politischökonomischer Ordnungen", Leviathan Sonderheft, vol. 40, núm. 27, 2012, pp. 9-39.

Nye, Joseph, "Globalization's democratic deficit: How to make international institutions more accountable", Foreign Affairs, vol. 80, núm. 2, 2001, pp. 2-6.

Offe, Clauss, "Wessen Wohl ist das Gemeinwohl?", en Harald Bluhm y Herfried Münkler, Gemeinwohl und Gemeinsinn: Rhetoriken und Perspektiven sozial-moralischer Orientierung, Berlín, Akademie Verlag, 2002.

Okereke, Chukwumerije, Harriet Bulkeley y Heike Schroeder, "Conceptualizing climate governance beyond international regime", Global Environmental Politics, vol. 9, núm. 1, 2009, pp. 58-78.

Ostroff, Joshua, "Daryl Hannah talks Keystone Pipeline, getting arrested, her new doc and Stephen Harper at sxsw", Huffington Post, 14 de marzo de 2014, en http:/ / www.huffingtonpost.ca

Partzsch, Lena, Global Governance in Partnerschaft: Die EU-Initiative "Water for Life", Baden-Baden, Nomos, 2007.

, "The power of celebrities in global politics", Celebrity Studies, vol. 6, núm. 2, 2015, pp. 178-191.

Prince, Rosa, "Leonardo DiCaprio and Sting among thousands on New York climate change march", The Telegraph, 2016, en www. telegraph.co.uk 
Richey, Lisa A. y Stefano Ponte, "Better (Red) ${ }^{\mathrm{TM}}$ than dead? Celebrities, consumption and international aid", Third World Quarterly, vol. 29, núm. 4, 2008, pp. 711-729.

_, "New actors and alliances in development", Third World Quarterly, vol. 35, núm. 1, 2014, pp. 1-21.

(ed.), Celebrity humanitarianism and North-South relations: Politics, place and power, Nueva York, Routledge, 2016.

y Alexandra C. Budabin, "Celebritizing conflict: How Ben Affleck sells the Congo to American", Humanity: An International Journal of Human Rights, Humanitarianism, and Development, vol. 7, núm. 1, 2016, pp. 27-46.

Scharpf, Fritz, Governing in Europe: Effective and democratic?, Oxford, University Press, 1999.

Schmitz, Björn y Volker Then, "Legitimation durch Narration Bindungskräfte durch das Erzählen von Geschichten" en Helga Hackenberg y Stefan Empter, Social Entrepreneurship - Social Business: Für die Gesellschaft unternehmen, Wiesbaden, VS Verlag für Sozialwissenschaften, 2011.

Sengers, F., R.P.J.M. Raven y A. Van Venrooij, "From riches to rags: Biofuels, media discourses, and resistance to sustainable energy technologies", Energy Policy, vol. 38, núm. 9, 2010, pp. 5013-5027.

Steffek, Jens, Claudia Kissling y Patrizia Nanz (eds.), Civil society participation in European and global governance: a cure for the democratic deficit?, Basingstoke, Palgrave MacMillan, 2008.

Stein, Tine, "Global social and civil entrepreneurs: An answer to the poor performance of global governance?", Wissenschaftszentrum Berlin, 2012, en www.bibliothek.wzb.eu

"Sustainable Biodiesel Alliance. Homepage", SBA, en www.sustainablebiodieselalliance.com

Take, Ingo, "Legitimes Regieren auf drei Ebenen - Konzeption und Analyseraster", en Ingo Take (ed.), Legitimes Regieren jenseits des Nationalstaates: Unterschiedliche Formen von Global Governance im Vergleich, Baden-Baden, Nomos, 2009.

"The World's Highest- Paid Celebrities", Forbes Magazine, en www. forbes.com 
Tsaliki, Liza, Christos Frangonikolopoulos y Asteris Huliaras (eds.), Transnational celebrity activism in global politics: Changing the world?, Chicago, University of Chicago Press, 2011.

Tucker, Karen, "Participation and subjectification in global governance: NGOs, acceptable subjectivities and the wTo", Millenium: Journal of International Studies, vol. 42, núm. 2, 2014, pp. 376-396.

Wheeler, Mark, Celebrity politics: image and identity in contemporary political communications, Cambridge, Polity Press, 2013.

Witte, Jan M. y Wolfgang Reinicke, Business as unusual: Facilitating United Nations reform through partnerships, Nueva York, United Nations Global Compact Office, 2005.

Wolf, Klaus D., "Contextualizing normative standards for legitimate governance beyond the state", en R. J. Grote y Bernard Gbiki (eds.), Participatory Governance. Political and Societal Implications, Opladen, Leske \& Budrich, 2002.

Ziegler, Jean, Report of the Special Rapporteur on the right to food: GE.0810098, 2008, en www.humanrights.ch/home/upload/pdf/08 0311_bericht_ziegler.pdf

Zürn, Michael, "Autorität und Legitimität in der postnationalen Konstellation”, Leviathan Sonderheft, vol. 40, núm. 27, 2012, pp. 41-62. 
